\title{
Crescimento relativo em Uca leptodactyla Rathbun (Crustacea Decapoda Ocypodidae) ${ }^{1}$
}

\author{
Setuko Masunari ${ }^{2} \&$ Bianca de Paula Swiech-Ayoub ${ }^{3}$
}

1 Contribuição número 1387 do Departamento de Zoologia, Universidade Federal do Paraná.

${ }^{2}$ Curso de Pós-graduação em Zoologia, Departamento de Zoologia, Universidade Federal do Paraná. Caixa Postal 19020, 81531-980 Curitiba, Paraná, Brasil.E-mail: setmas@bio.ufpr.br

${ }^{3}$ Centro Universitário Campos de Andrade. Rua João Negrão 1285, 80230-150 Curitiba, Paraná, Brasil.

\begin{abstract}
Relative growth in the fiddler crab Uca leptodactyla Rathbun (Crustacea Decapoda Ocypodidae). Relative growth of the male major chela and female abdome was studied in a population of the fiddler crab Uca leptodactyla Rathbun, 1898 from Itapoá, Santa Catarina coast, southern Brazil. Major chela length (CMQ) was measured from 191 males, and abdomen width (LAB) from 128 females. Carapace width (LC) was the reference dimension for both sexes, which ranged from 3.9 to $11.5 \mathrm{~mm}$ for males, and from 3.15 to $10.65 \mathrm{~mm}$ for females. Males grew bigger than females. Relationship between CMQ and LC showed a transition point at $8.35 \mathrm{~mm}$ LC in males, and between LA and LC at $7.10 \mathrm{~mm}$ LC in females. Growth was allometrically positive in the early ontogenesis and isometric after the puberal molting for both sexes. Regressions between LC and CMQ in males read as: $\log C M Q=-0,854536+2,19 . \operatorname{logLC}$ for empirical points at left of critical point and $\log C M Q=$ $0,063047+1,24 . \log L C$ for those at right. In females, this relation was $\log L A B=-0,603590+1,30 . \log L C$ and $\log L A B=-0,361464+1,07 . \log L C$, respectively. These body dimensions were connected with reproductive activity of this species.
\end{abstract}

KEY WORDS. Abdomen width, major chela.

O gênero Uca Leach, 1814 é constituído de, pelo menos, 62 espécies que têm distribuição em substratos lamosos do litoral dos trópicos, quase sempre associados a manguezais. Entretanto, algumas espécies podem ocorrer em latitudes maiores como no Japão e na Nova Inglaterra, Austrália. São animais freqüentemente simpátricos, sempre gregários, de hábito diurno e ativos durante a maré baixa (Crane 1975). Melo (1996) cita 10 espécies de Uca para o litoral brasileiro das quais cinco ocorrem no litoral do Estado de Santa Catarina: Uca leptodactyla Rathbun, 1898, Uca rapax (Smith, 1870), Uca thayeri Rathbun, 1900, Uca uruguayensis Nobili, 1901 e Uca vocator (Herbst, 1804).

Estes caranguejos caracterizam-se pela presença de uma enorme quela num dos quelípodos do macho, tendo a quela do outro quelípodo um tamanho diminuto. Em contraste, as quelas de ambos os quelípodos da fêmea são diminutas. A quela gigante tem importante papel nos comportamentos agonísticos e de corte nupcial (CRANE 1975), a qual se desenvolve nos machos no período de maturação sexual: ela não está presente nos primeiros estágios de desenvolvimento dos mesmos (YAMAGUCHI 1977). Esta característica nos machos conferiu a estes animais o nome popular de "caranguejo-violinistas" nos países de língua inglesa, uma alusão ao comportamento destes animais de movimentar ritmicamente a maior quela para a frente e para trás ("waving"), lembrando um violinista executando uma música. No Brasil, eles são conhecidos como "chamamarés", onde o movimento é interpretado como um aceno para provocar a subida da maré.

Dá-se o nome de crescimento relativo quando certas dimensões do corpo de um animal modificam-se em taxas diferentes de outras, o que leva a mudanças nas proporções com o aumento do tamanho. Certas alterações podem ser bruscas e refletem um estágio crítico ao longo da ontogenia como muda pré-puberal ou puberal (HARTNOLL 1978, 1982). Um gráfico de dispersão dos pontos empíricos de duas dimensões do corpo pode mostrar estas alterações bruscas através dos pontos de inflexão. Esta metodologia tem sido utilizada, também, para detectar crescimento relativo em populações de crustáceos que se desenvolvem em diferentes condições de precipitação pluviométrica (MarTinez-MaYén et al. 2000), e naquelas que migram de um hábitat para o outro durante o seu desenvolvimento (КоваYASH 2002).

Apesar da abundância de Uca leptodactyla no litoral do Estado de Santa Catarina, não há estudos sobre o crescimento relativo desta espécie. Entretanto, muitos trabalhos foram pu- 
blicados acerca do crescimento relativo em Brachyura com o objetivo de relacionar o crescimento da carapaça, quela e abdome com a muda puberal, que marca o início da maturidade sexual das espécies. No Brasil, podem-se citar os estudos realizados no litoral paulista com os Brachyura Arenaeus cribrarius (Lamarck, 1818) por Pinheiro \& Fransozo (1993), Portunus spinimanus Latreille, 1819 por SANTos et al. (1995), Goniopsis cruentata (Latreille, 1803) por Сово \& Fransozo (1998) e Callinectes ornatus Ordway, 1863 por Mantelatto \& Martinelli (1999). Para o litoral do Estado do Paraná, conhece-se somente o trabalho de Kowalczuk \& Masunari (2000) sobre o crescimento relativo de Armases angustipes (Dana, 1852) proveniente da Ilha do Farol, Matinhos. Do estrangeiro, citam-se os trabalhos de Abello et al. (1990) sobre as espécies Liocarcinus depurator (Linnaeus, 1758) e Macropipus tuberculatus (Roux, 1830) coletados na Espanha, de CARSEN et al. (1996) sobre Platyxanthus patagonicus A. Milne Edwards, 1879 coletado na Patagônia, Argentina, de CHu (1999) sobre Charybdis affinis Dana, 1852 da China, de Tsuchida \& Fujikura (2000) sobre Austinogrea williamsi Hessler \& Martin, 1989 do Jара̃o e de Котв \& HarTnOLl (2002) sobre Hapalocarcinus marsupialis Stimpson, 1859 do Mar Vermelho, dentre outros. Sobre o gênero Uca, Rosenberg (2000) publicou um estudo morfométrico sobre as quelas, analisando variáveis intra e interespecíficas.

O presente trabalho visa descrever o crescimento relativo da maior quela do macho e do abdome da fêmea de Uca leptodactyla, em relação à largura da carapaça; estas variáveis estão associadas aos padrões reprodutivos dos crustáceos.

\section{MATERIAL E MÉTODOS}

A área de estudo localiza-se em Itapoá, no litoral de Santa Catarina, à margem direita do Rio Saí-Guaçu, na altura de sua desembocadura para o mar $\left(25^{\circ} 36^{\prime} 00^{\prime \prime} \mathrm{S}\right.$ e $\left.48^{\circ} 33^{\prime} 20^{\prime \prime} \mathrm{W}\right)$. Nessa área, ocorre um extenso banco de areia, onde crescem marismas do gênero Spartina Schreb que formam manchas na paisagem arenosa. Os animais foram coletados manualmente, com auxílio de pás de jardim, durante as marés de sizígia, nos meses de junho e julho de 1999. Os caranguejos coletados foram anestesiados em sacos plásticos contendo um chumaço de algodão embebido em éter, para evitar que os animais perdessem os quelípodos ao interagirem uns com os outros. No laboratório, após fixação e conservação, os caranguejos tiveram o sexo reconhecido e a largura da carapaça (LC) medida com um paquímetro de precisão de $0,01 \mathrm{~mm}$. Para animais menores, as medidas foram tomadas sob microscópio estereoscópico com ocular graduada. Os machos, ainda tiveram o comprimento da maior quela (CMQ) medido, da extremidade proximal do própodo até a ponta do dedo fixo. Das fêmeas foi medida a largura do abdome (LAB), no ponto de máxima. Estas duas dimensões foram escolhidas porque estão relacionadas com a atividade reprodutiva de cada sexo. Os pontos de inflexão nos gráficos de dispersão foram calculados com o programa para microcomputadores REGRANS desenvolvido por Pezzuto (1993). A variável largura da carapaça (LC) foi utilizada como variável independente $(\mathrm{X})$ por ser a medida de maior amplitude, contra a qual outras foram testadas $(\mathrm{Y})$. Foi utilizada a transformação $\operatorname{logarítmica~} \log \mathrm{Y}=\log \mathrm{a}+\mathrm{b} \cdot \log \mathrm{X}$ da função exponencial $\mathrm{Y}=\mathrm{a} \mathrm{X}^{\mathrm{b}}$. Os valores de $\mathrm{a}$ e $\mathrm{b}$ foram obtidos pela determinação da regressão de $\log Y$ e $\log X$ usando o método dos mínimos quadrados, o qual permite a aplicação de testes sobre a declividade (Huber 1985). O valor de b é a constante de alometria. Valor de $\mathrm{b}=1$ indica crescimento isométrico, $\mathrm{b}>1$ crescimento alométrico positivo e $\mathrm{b}<1$ crescimento alométrico negativo. Para verificar alometria ou isometria no crescimento, utilizou-se o teste-t de Student.

O ponto de inflexão foi testado através da comparação da soma dos quadrados dos resíduos em Y (SSRy) da reta total e da somatória da SSRy à direita e à esquerda do ponto de inflexão segundo SOMERTON (1980). Os intervalos de confiança para os valores da declividade foram calculados com base em SOKAL \& ROHLF (1969).

\section{RESULTADOS}

Foi analisado um total de 319 caranguejos, dos quais, 191 machos e 128 fêmeas. A LC dos machos variou de 3,9 a11,5 mm enquanto a das fêmeas, de 3,15 a 10,65 mm; o CMQ dos machos variou de 2,1 a 23,3 mm e a LA das fêmeas, de 1,05 a $5,45 \mathrm{~mm}$.

Na ontogênese dos machos, o CMQ mostrou um ponto de inflexão em $8,35 \mathrm{~mm} \mathrm{LC}$, tendo o subconjunto à esquerda no gráfico de dispersão dos pontos empíricos 128 indivíduos e no subconjunto à direita, 63 indivíduos (Fig. 1) (SQD em $\mathrm{Y}=\mathrm{F}(3,04)=7,71, \mathrm{a}=5 \%)$. Os pontos à esquerda do ponto de inflexão mostraram um crescimento alométrico positivo (teste de significância da declividade $-\mathrm{TSD}=\mathrm{t}(1,98)=15,15$, $\alpha=5 \%)$. Assim, em machos juvenis, o CMQ cresce mais que a LC. A expressão que determina a relação entre LC e CMQ em machos juvenis é a seguinte: $\log C M Q=-0,854536+2,19 . \operatorname{logLC}$.

Por outro lado, os pontos à direita do ponto de inflexão refletem um crescimento isométrico $(\mathrm{TSD}=\mathrm{t}(2,00)=1,64$, $\alpha=5 \%$ ), com a seguinte equação da reta para machos adultos: $\log \mathrm{CMQ}=0,063047+1,24 . \log \mathrm{L}$.

No gráfico de dispersão dos pontos empíricos, é possível verificar que o CMQ tem valor menor do que a LC entre os caranguejos com LC menor do que $6,0 \mathrm{~mm}$. Após este valor, o CMQ passa a ter valor maior do que a própria LC.

Dentre as fêmeas, o crescimento da largura do abdome foi alométrico positivo no início do desenvolvimento e após a muda puberal, tornou-se isométrico. O gráfico de dispersão dos pontos empíricos entre a LC e a LAB mostrou um ponto de inflexão em 7,40 mm LC, tendo o subconjunto à esquerda no gráfico de dispersão dos pontos empíricos 70 indivíduos e no subconjunto à direita, 58 indivíduos (Fig. 2) (SQD em $\mathrm{Y}=\mathrm{F}(3,09)=57,45, \mathrm{a}=$ $5 \%$ ). Os pontos à esquerda do ponto de inflexão mostraram um crescimento alométrico positivo (TSD $=\mathrm{t}(2,00)=5,88, \mathrm{a}=5 \%$ ), onde a LAB cresce mais que a LC em fêmeas juvenis. A expressão que determina a relação entre LC e LAB em fêmeas juvenis é a seguinte: $\log \mathrm{LAB}=-0,603590+1,30 \cdot \log \mathrm{LC}$.

Por outro lado, os pontos à direita do ponto de inflexão refletem um crescimento isométrico entre LC e LAB de fêmeas adultas (TSD $=\mathrm{t}(2,00)=0,68, \mathrm{a}=5 \%)$, com a seguinte equação da reta: $\log \mathrm{LAB}=-0,361464+1,07 . \log \mathrm{LC}$.

A tabela I mostra a estatística das regressões lineares das dimensões estudadas em machos e fêmeas.

\section{DISCUSSÃO}

Já está bem estabelecido na literatura, que em Crustacea Brachyura, as variáveis morfométricas mais apropriadas para estimar o tamanho do início da maturidade sexual em machos e fêmeas são as medidas do quelípodo e do abdome respectiva- 

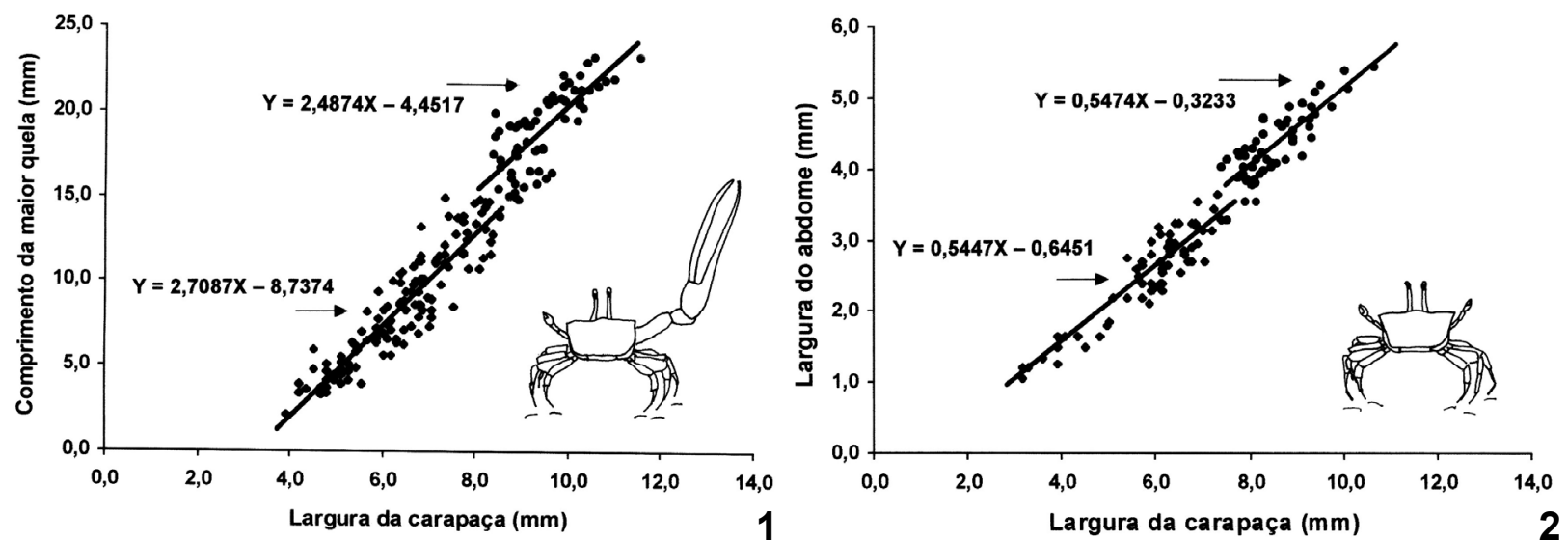

Figuras 1-2. Uca leptocdactyla. Dispersão dos pontos empíricos da relação entre a largura da carapaça (LC) e o comprimento da maior quela (CMQ) dos machos (1) e entre a largura da carapaça (LC) e a largura do abdome (LA) das fêmeas (2).

Tabela I. Uca leptodactyla. Estatística das regressões lineares entre a largura da carapaça (LC) e o comprimento da maior quela (CMQ) dos machos e entre a largura da carapaça (LC) e a largura do abdome (LAB) das fêmeas. (a) intersecção, (b) declividade, (N) tamanho da amostra, (r) coeficiente de correlação, $\left(\mathrm{R}^{2}\right)$ coeficiente de determinação, (X) Ponto de inflexão.

\begin{tabular}{lccccccc}
\hline & Variáveis & $\mathrm{N}$ & $\mathrm{r}$ & $\mathrm{R}^{2}$ & Log a & b (I.C. 95\%) & Alometria \\
\hline \multirow{2}{*}{ Machos (LC X CMQ X = 8,35) } & $<\mathrm{X}$ & 128 & 0,92153 & 0,849209 & 0,854536 & $2,19(2,04-2,35)$ & + \\
& $=\mathrm{X}$ & 63 & 0,72989 & 0,532736 & 0,063047 & $1,24(0,95-1,54)$ & 0 \\
Fêmeas (LC X LAB, X = 7,40) & $<\mathrm{X}$ & 70 & 0,94953 & 0,901604 & 0,603590 & $1,30(1,20-1,41)$ & + \\
& $=\mathrm{X}$ & 58 & 0,80463 & 0,647427 & 0,361464 & $1,07(0,86-1,28)$ & 0 \\
\hline
\end{tabular}

mente (Pinheiro \& Fransozo 1993). As quelas são maiores nos machos e o abdome é mais largo nas fêmeas. Essas características sexuais secundárias estão relacionadas com diferentes papéis reprodutivos dos dois sexos (Tsuchida \& FujIKURA 2000). Nos machos, uma quela relativamente grande aumenta o seu tamanho aparente que passa a ter importância nos encontros agonísticos, defesa, captura de alimento, demarcação de território e na copulação. Para as fêmeas, um abdome relativamente grande também é importante na perpetuação da espécie, pois permite proteger e incubar ovos e algumas vezes juvenis recém-eclodidos. Portanto, o significado adaptativo dessas estruturas é evidente.

O modelo padrão proposto por HaRTNOLL (1974) evidencia uma alometria positiva considerável no crescimento da maior quela do macho, tanto na fase pré-puberal como na puberal. Inúmeros autores encontraram esse modelo proposto em espécies variadas de Brachyura de locais distintos, a saber, em Arenaeus cribrarius, Portunus spinimanus e Callinectes ornatus provenientes de Ubatuba, SP (Pinheiro \& Fransozo 1993, Santos et al. 1995, Mantelatto \& Martinelli 1999), e em Armases angustipes do litoral do Estado do Paraná (KowalczuK \& MASUNARI 2000). Do estrangeiro, este padrão foi descrito para Potamon potamios palestinensis da região do Mar Morto (GHERARDI \& Micheli 1989), para Goneplax rhomboides, Liocarcinus depurator e Macropipus tuberculatus e provenientes do litoral da Espanha (Abello \& Sarda 1982, Abello et al. 1990).
Para os machos de Uca leptodactyla do presente trabalho, o ponto de inflexão detectado em $8,35 \mathrm{~mm}$ LC está relacionado com a maturidade sexual e, provavelmente, a muda puberal deve ocorrer quando a LC chega próxima a este valor. Os indivíduos com LC inferior a $8,35 \mathrm{~mm}$ são juvenis e o crescimento alométrico positivo do CMQ indica que a maior quela cresce proporcionalmente mais rápido do que a LC. Por outro lado, nos machos maturos sexualmente, o crescimento isométrico entre LC e CMQ mostra que ambas as variáveis crescem na mesma proporção.

Em algumas das publicações acima referidas, os autores analisaram em separado o crescimento das quelas em juvenis e adultos. Nestes estudos foi observado crescimento alométrico positivo em ambos os estágios, em maior ou menor intensidade. Entretanto, a quela dos machos de $U$. leptodactyla na sua fase adulta mostrou um crescimento isométrico, o que parece não ser uma regra dentre os Decapoda Brachyura. Esta característica pode estar relacionada com a necessidade dos machos manterem o equilíbrio do corpo, pois, a maior quela atinge um comprimento até duas vezes maior do que a própria largura da carapaça nesta espécie. Portanto, um crescimento alométrico positivo após a muda puberal resultaria numa quela desproporcionalmente pesada e longa, comprometendo o equilíbrio do animal, especialmente durante a execução do "waving", o padrão comportamental típico do período reprodutivo destes chama-marés (Crane 1975). 
Dentre as fêmeas de $U$. leptodactyla, observou-se que nas imaturas, a largura do abdome aumenta mais que a largura da carapaça, sendo o crescimento do abdome alométrico positivo. Porém, após a maturidade sexual, nas fêmeas adultas, a largura do abdome e da carapaça crescem na mesma proporção, sendo o crescimento isométrico.

O crescimento alométrico positivo da largura da carapaça em fêmeas de Brachyura é descrito para Portunus spinimanus, Platyxanthus patagonicus, Calinectes ornatus, Armases angustipes, Austinograea williamsi (SANTOS et al. 1995, CARSEN et al. 1996, Mantelatto \& Martinelli 1999, Kowalczuk \& Masunari 2000, TSUCHIDA \& FUJIKURA 2000). Entretanto, não há referências sobre crescimento isométrico em fêmeas adultas. Portanto, esta constatação, também, foge do padrão observado para muitas espécies cujo crescimento relativo é conhecido. Isso pode ser resultado da utilização de apenas uma equação para expressar o crescimento relativo total do abdome das fêmeas. Entretanto, alguns pesquisadores que fizeram análise de crescimento relativo em cada estágio de desenvolvimento, sempre obtiveram crescimento alométrico positivo do abdome, mesmo após a muda puberal. Por exemplo, Cobo \& Fransozo (1998), ao analisarem o crescimento relativo do abdome de fêmeas obtiveram dois pontos de inflexão, e separaram a população de fêmeas de Goniopsis cruentata em juvenis, pré-púberes e adultas; nas três subpopulações, o crescimento do abdome foi alométrico positivo, embora nas fêmeas pré-púberes, a alometria tenha sido mais marcante. No Canadá, ao analisarem o crescimento relativo do abdome de fêmeas de Chionoecetes opilio (O. Fabricius, 1780), AlunNo-Bruscia \& SAINTE-Marie (1998) obtiveram um resultado bastante parecido, onde as fêmeas foram divididas em três subpopulações com as mesmas características de crescimento alométrico positivo, tendo sido as fêmeas prépúberes mostrado uma alometria positiva também mais evidente. GHERARDi \& Micheli (1989), ao estudarem a estrutura populacional de Potamon potamios palestinensis no Mar Morto observaram crescimento alométrico positivo do abdome de fêmeas antes e após a maturação sexual, embora a alometria tenha diminuído após a muda puberal. O mesmo resultado foi obtido por Pinheiro \& Fransozo (1993) em fêmeas de Arenaeus cribrarius em Ubatuba. CHU (1999) cita que o abdome de fêmeas de Charybdis affinis na China, cresce até a puberdade. De um modo geral, embora os diversos estudos revelem crescimento alométrico positivo do abdome em fêmeas, a alometria diminui na fase adulta. Dessa forma, o crescimento relativo do abdome de fêmeas de $U$. leptodactyla não difere muito do crescimento das outras espécies de Brachyura e, a constatação da isometria pode ser considerada como um refinamento na análise. Pode-se inferir que boa parte da energia é alocada para produzir e carregar a massa ovígera após a muda da puberdade, quando o abdome já foi alargado o suficiente antes da referida muda.

O crescimento relativo observado em ambos os sexos de $U$. leptodactyla, capacita e prepara estes animais para a vida reprodutiva, resultando numa adaptação das quelas nos machos para as interações agonísticas e de "waving" e do abdome das fêmeas para abrigar a massa ovígera.

\section{AGRADECIMENTOS}

Aos acadêmicos Daniella Gobbo Bordon e Alberto Barcellos, ambos bolsistas do Programa de Iniciação Científica PIBIC/CNPq da Universidade Federal do Paraná, pelo auxílio na obtenção de medidas morfométricas. Aos dirigentes desta instituição pela concessão das bolsas.

\section{REFERÊNCIAS BIBLIOGRÁFICAS}

Abello, P.; J.P. Pertierra \& D.G. Reid. 1990. Sexual size dimorphism, relative growth and handedness in Liocarcinus depurator and Macropipus tuberculatus (Brachyura: Portunidae). Scientia Marina, Barcelona, 54 (2):195-202.

Abello, P. \& F. SARDA. 1982. Morphometric characters of the sexual dimorphism in the chelae of Goneplax rhomboides (L.) (Decapoda: Brachyura). Investigacion Pesquera, Barcelona, 46 (2): 163-170.

Alunno-Bruscia, M. \& B. Sainte-Marie. 1998. Abdomen allometry, ovary development, and growth of female snow crab, Chionoecetes opilio (Brachyura, Majidae), in the Northwestern Gulf of St. Lawrence. Canadian Journal of Fisheries and Aquatic Sciences, Ottawa, 55 (2): 459-477.

Carsen, A.E.; S. Kleinman \& M.A. Scelzo. 1996. Fecundity and relative growth of the crab Platyxanthus patagonicus (Brachyura: Platyxanthidae) in Patagonia, Argentina. Journal of Crustacean Biology, Lawrence, 16 (4): 748-753.

$\mathrm{CHU}$, K.H. 1999. Morphometric analysis and reproductive biology of the crab Charybdis affinis (Decapada, Brachyura, Portunidae) from the Zhujiang estuary, China. Crustaceana, Leiden, 72 (7): 647-658.

Сoвo, V.J. \& A. Fransozo. 1998. Relative growth of Goniopsis cruentata (Crustacea, Brachyura, Grapsidae), on the Ubatuba region, São Paulo, Brazil. Iheringia, Série Zoologia, Porto Alegre, (84): 21-28.

Crane, J. 1975. Fiddler crabs of the world. Princeton, Princeton University Press, 736p.

Gherardi, F. \& F. Micheli. 1989. Relative growth and population structure of the freshwater crab, Potamon potamios palestinensis, in the Dead Sea area (Israel) Israel Journal of Zoology, Jerusalem, 36 (3-4): 133-145.

HaRTNOLL, R.G. 1974. Variation in growth patterns between some secondary sexual characters in crabs (Decapoda: Brachyura). Crustaceana, Leiden, 27: 131-136.

1978. The determination of relative growth in Crustacea. Crustaceana, Leiden, 34 (3): 281-289.

1982. Growth, p. 111-196. In: L.G. Abele (Ed.). The biology of Crustacea. New York, Academic Press, vol. 2, $\mathrm{XX}+440 \mathrm{p}$.

Huber, M.E. 1985. Allometric growth of the carapace in Trapezia (Brachyura, Xanthidae). Journal of Crustacean Biology, Lawrence, 5 (1): 79-83.

Kobayashi, S. 2002. Relative growth pattern of walking legs of the Japanese mitten crab Eriocheir japonica. Journal of Crustacean Biology, Lawrence, 22 (3): 601-606.

Котв, M.M.A. \& R.G. Hartnoll. 2002. Aspects of the growth and reproduction of the coral gall crab Hapalocarcinus marsupialis. Journal of Crustacean Biology, Lawrence, 22 (3): 558-566.

Kowalczuk, V.G.L. \& S. Masunari. 2000. Crescimento relativo e determinação da idade na fase juvenil de Armases angustipes (Dana) (Decapoda, Brachyura, Grapsidae). Revista Brasileira de Zoologia, Curitiba, 17 (1): 17-24.

Mantelatto, F.L.M. \& J.M. Martinelli. 1999. Carapace widthweight relationships of Callinectes ornatus (Brachyura, 
Portunidae) from Ubatuba Bay, Brazil. Iheringia, Série Zoologia, Porto Alegre, (87): 111-116.

Martínez-Mayén, M.; R. Román-Contreras; A. Rocha-Ramírez \& S. Chazaro-Olvera. 2000. Relative growth of Atya margarita A. Milne-Edwards, 1864 (Decapoda, Atyidae) from the southern Pacific coast of Mexico. Crustaceana, Leiden, 73 (5): 525-534.

MeLo, G.A.S. 1996. Manual de identificação dos Brachyura (caranguejos e siris) do litoral brasileiro. São Paulo, Plêiade/FAPESP, 604p.

Pezzuto, P.R. 1993. Regrans: a "basic" program for an extensive analysis of relative growth. Atlântica, Rio Grande, 15: 93105.

Pinheiro, M.A.A. \& A. Fransozo. 1993. Relative growth of the speckled swimming crab Arenaeus cribrarius (Lamarck, 1818) (Brachyura, Portunidae), near Ubatuba, State of São Paulo, Brazil. Crustaceana, Leiden, 65 (3): 365-376.

Rosenberg, M.S. 2002. Fiddler crab claw shape variation: a geometric morphometric analysis across the genus Uca (Crusta- cea: Brachyura: Ocypodidae). Biological Journal of the Linnean Society, London, 75 (2): 147-162.

Santos, S.; M.L. Negreiros-Fransozo \& A. Fransozo. 1995. Morphometric relationships and maturation in Portunus spinimanus Latreille, 1819 (Crustacea, Brachyura, Portunidae). Revista Brasileira de Biologia, Rio de Janeiro, 55 (4): 545-553.

SOKAL, R.R. \& F.J. RoHLF. 1969. Biometry. The principles and practice of statistics in biological research. San Francisco, Freeman, 776p.

SomerTon, D.A. 1980. A computer technique for estimating the size of sexual maturity in crabs. Canadian Journal of Fisheries and Aquatic Sciences, Ottawa, 37: 1488-1494.

Tsuchida, S. \& K. Fujikura. 2000. Heterochely, relative growth and gonopod morphology in the bythograeid crab, Austinograea williamsi (Decapoda, Brachyura). Journal of Crustacean Biology, Lawrence, 20 (2): 407-414.

YAMAGUCHI, T. 1977. Studies on the handedness of the fiddler crab Uca lactea. Biological Bulletin, Woods Hole, 152: 424436.

Recebido em 21.X.2002; aceito em 21.VIII.2003. 